\title{
Trends in cutaneous sensitization in the first 18 years of life: results from the 1989 Isle of Wight birth cohort study
}

\author{
G. Roberts ${ }^{1,2}$, H. Zhang ${ }^{3}$, W. Karmaus ${ }^{3}$, A. Raza ${ }^{1,2}$, M. Scott ${ }^{1,2}$, S. Matthews ${ }^{1}$, R. J. Kurukulaaratchy ${ }^{1,2}$, T. Dean $^{1,4}$ and $^{1}$ \\ S. H. Arshad ${ }^{1,2}$ \\ ${ }^{1}$ The David Hide Asthma and Allergy Research Centre, St Mary's Hospital, Newport, UK, ${ }^{2}$ University of Southampton Faculty of Medicine, Southampton, \\ UK, ${ }^{3}$ Department of Epidemiology and Biostatistics, Norman J. Arnold School of Public Health, University of South Carolina, Columbia, SC, USA and \\ ${ }^{4}$ University of Portsmouth, Portsmouth, UK
}

\section{Clinical Et Experimental Allergy}

Correspondence:

Graham Roberts, The David Hide

Asthma and Allergy Research Centre,

St Mary's Hospital, Newport, Isle of

Wight, P030 5TG, UK.

E-mail: g.c.roberts@southampton.ac. uk

Cite this as: G. Roberts, H. Zhang, W. Karmaus, A. Raza, M. Scott,

S. Matthews, R. J. Kurukulaaratchy,

T. Dean and S. H. Arshad, Clinical \&t

Experimental Allergy, 2012 (42) 15011509.

\begin{abstract}
Summary
Background Skin prick testing (SPT) is fundamental to the practice of clinical allergy identifying relevant allergens and predicting the clinical expression of disease. There are only limited data on the natural history of SPT results over childhood and adolescence. Objective We aimed to describe the natural history of SPT and patterns of sensitization over childhood and adolescence.

Methods The 1989 Isle of Wight birth cohort (1456 participants) was followed up at 1, 2, 4, 10 and 18 years. SPT was undertaken from 4 years.

Results SPT was performed on 980 (80\%), 1036 (75\%) and 853 (65\%) of participants at 4, 10 and 18 years. The prevalence of sensitization to any allergen at these time-points was $19.7 \%, 26.9 \%$ and $41.3 \%$ respectively. At each time-point, boys were significantly more likely to be sensitized $(P<0.016)$ and sensitization significantly increased over childhood and adolescence (average annual increase of 7\%). Some children outgrew their sensitization. The rate of sensitization to most individual allergens increased over childhood and adolescence. A configural frequency analysis showed that whether an individual was sensitizated was relatively fixed over childhood and adolescence. Cluster analysis at 4 years demonstrated four major groups of individuals with similar co-sensitization to specific allergens. Children who were sensitized at age 4 years generally went onto become sensitized to additional allergens at 10 and 18 years.

Conclusions and Clinical Relevance Allergic sensitization continues to increase over childhood into adolescence although the majority of children who were not sensitized at 4 years remain non-sensitized throughout childhood and adolescence. The presence of sensitization at 4 years predicted later sensitization to additional allergens.
\end{abstract}

Keywords atopy, adolescence, childhood, prevalence, sensitization, allergy Submitted 18 March 2012; revised 03 June 2012; accepted 12 June 2012

\section{Introduction}

Clinical allergy results from the generation of specific IgE against common environmental proteins. Allergic diseases pose important health care problems and are associated with significant morbidity [1-4]. In clinical practice, atopy is defined as the presence of sensitization to allergen by skin prick testing (SPT) or by the presence of serum-specific IgE [5]. Atopy is regarded as a condition that generally develops early in childhood with increased levels of cord blood total IgE preceding its development in some, but not all, children [1, 6, 7].
The presence of atopy has a major influence on the development and course of common childhood conditions such as asthma [8-10] and rhinitis [11]. Atopy is generally regarded as an all or nothing condition although the magnitude of the specific IgE or SPT response is related to the likelihood of both allergic airway disease [12] and food allergy [13,14]. In addition, larger magnitudes are associated with higher levels of inflammatory markers [15] and a more severe expression of allergic diseases such as asthma [16]. Atopy has been thought of as a stable condition with little appreciable remission; this is now being questioned [17]. 
There are only limited data on the natural history of sensitization through childhood and adolescence [18-20]. Much of this relates to serum-specific IgE levels [20] rather than cutaneous sensitization although SPT is more widely used in clinical practice. Other groups have compared different cohorts focusing on whether rates are changing with passing time rather than increasing age [17]. The 1989 Isle of Wight birth cohort has been followed up from birth to 18 years of age $[1,15,21-23]$. Previous analysis of this cohort has assessed the pattern of allergic sensitization during the first decade of life [1]. In this article, we examine the natural history of cutaneous sensitization over the first 18 years of life to assess how patterns of allergic sensitization change through childhood and adolescence.

\section{Methods}

\section{Participants and assessments}

An unselected, whole population birth cohort was established on the Isle of Wight, United Kingdom, to prospectively study the natural history of allergic disorders. The Island is close to the British mainland, semi-rural, with no heavy industry and a good mix of social classes. The population is $99 \%$ white. Of the 1536 babies born between January 1989 and February 1990, we were able to follow up 1456 infants from age 1 year onwards [21-23]. Participants were assessed at $1,2,4,10$ and 18 years. Detailed questionnaires were completed in person, by telephone or post. SPT was undertaken in a standardized manner to a standard battery of common allergens (house dust mite, cat, dog, Alternaria alternata, Cladosporium herbarium, grass pollen mix, and tree pollen mix, cows' milk, soya, hens' egg, peanut and cod plus positive and negative controls; ALK, Horsholm, Denmark) at 4, 10 and 18 years on most participants attending the research centre. Standard lancets (ALK) were used with the average of the longest diameter and the diameter perpendicular to it being assessed at $15 \mathrm{~min}$ [5]. Results were discounted if the positive control was not at least $3 \mathrm{~mm}$ in diameter. Allergic sensitization to an allergen was defined as a mean weal diameter of $3 \mathrm{~mm}$ greater than the negative control with atopy defined by having a positive SPT to at least one allergen. The local Research Ethics Committee approved this study (06/Q1701/34).

\section{Statistical analyses}

For each study period, point prevalence for sensitization was determined for each allergen available in our database. The prevalence was calculated by dividing the number of participants with sensitization to an allergen by the total number of participants who had available information regarding that specific allergen. The rates of positive and negative transitions of sensitization to any allergen were investigated. The positive transition between two study time-points was defined as a change in status from non-sensitized to sensitized. The negative transition between study time-points was defined as a change from sensitized to a non-sensitized status.

For each observation time-point (age 4, 10, 18 years), we estimated the difference of the prevalence in males and females using one-sample $z$-test on proportions. To further assess the trend of prevalence across age, we conducted a longitudinal analysis using repeated measures of sensitization at different ages. Log-linear models with repeated measures were fitted to assess the average number of sensitized participants. Generalized estimated equation (GEE) analysis [24] taking the within-child effect into account was applied using the GENMOD procedure in the SAS system (SAS, Gary, USA). Experiment-wise significance level was set at 0.05 . We estimated marginal prevalence trend across time, between sex and their interaction given that our preliminary analysis demonstrated that prevalence of sensitization varied by age. To evaluate the positive and negative transition rates and their trends over time, the rates of change were calculated and the significance of the change was evaluated based on two-sided, twosample proportion tests.

To further understand how sensitization changes across age at the level of individuals children, we performed a pattern analysis using a configural frequency test [25]. Configural frequency analysis assesses the statistical chance that the observed pattern (e.g. pattern 'NNP' means negative SPT results at ages 4 and 10 followed by a positive result at age 18 years) is different from the pattern that is most likely to occur by chance. This analysis was applied to sensitization to indoor and outdoor allergens. The Bonferroni method was applied to keep experiment-wise type I error at or below the 0.05 level.

To understand the pattern of sensitization at the level of specific allergens over time, we applied cluster analyses at each assessment. This assigns participants with similar SPT results at a specific time-point to the same cluster thereby unmasking patterns in the dataset. First, at each assessment point, two clusters were first manually formed containing the participants with missing data and second those who were not sensitized. The cluster method of k-means was then applied to the remaining data in which every child had at least one positive SPT to describe patterns of sensitization. At each assessment time $(4,10,18$ years), the number of clusters was determined. 


\section{Results}

\section{Participants and sensitization at each assessment}

A total of 1218 (84\%), 1373 (94\%) and 1313 (90\%) participants had clinical information available at age 4,10 and 18 years respectively. SPT was performed on 980 (80\%), 1036 (75\%) and 853 (65\%) of these participants at each time-point (Table 1). The average (SD) weal sizes to the histamine positive controls were $3.5 \mathrm{~mm}$ (1.2), $4.8 \mathrm{~mm}$ (1.3) and $5.3 \mathrm{~mm}$ (1.3) at each time-point. There was no obvious selection bias in terms of the presence of asthma, eczema or rhinitis for SPT assessment. Significantly, more females (68.0 vs. 62.3\%, $P=0.002$ ) were assessed at age 18 years (Table 1 ), but there was no relationship between proportion of followup and SPT results for all participant or when split by gender (data not shown). There was a strong association between a positive SPT at any age and the presence of asthma, eczema and rhinitis at that assessment (Table 2).

\section{Prevalence of sensitization across childhood and adolescence}

A general trend was observed of increasing prevalence of sensitization with age (Table 3). This was particularly true for the aeroallergens and peanut. The exceptions were egg, milk and soya which demonstrated no significant change in the prevalence of sensitization. Males

Table 1. Comparison of subjects who were skin prick tested at each assessment

\begin{tabular}{|c|c|c|c|c|}
\hline & & 4 years $n(\%)$ & 10 years $n(\%)$ & 18 years $n(\%)$ \\
\hline \multicolumn{5}{|c|}{ SPT available } \\
\hline All & & $980(80.5 \%)$ & $1036(75.5 \%)$ & $853(65.0 \%)$ \\
\hline Males & & $493(79.5 \%)$ & $516(74.0 \%)$ & $407(62.3 \%)$ \\
\hline Females & & $487(81.6 \%)$ & $520(76.9 \%)$ & $446(68.0 \%)$ \\
\hline$P$-value & & 0.37 & 0.12 & 0.002 \\
\hline \multicolumn{5}{|c|}{ SPT available by asthma, eczema or rhinitis } \\
\hline \multirow[t]{2}{*}{ Asthma } & SPT & $71(44.1 \%)$ & $100(70.0 \%)$ & $114(69.9 \%)$ \\
\hline & No SPT & $90(55.9 \%)$ & $64(30.0 \%)$ & $49(30.1 \%)$ \\
\hline \multirow[t]{2}{*}{ Eczema } & SPT & $53(42.7 \%)$ & $61(38.4 \%)$ & $56(53.3 \%)$ \\
\hline & No SPT & $71(57.3 \%)$ & $98(61.6 \%)$ & $49(46.7 \%)$ \\
\hline \multirow[t]{2}{*}{ Rhinitis } & SPT & $33(55.0 \%)$ & $138(51.3 \%)$ & $233(69.8 \%)$ \\
\hline & No SPT & 27 (45.0\%) & $131(48.7 \%)$ & $101(30.2 \%)$ \\
\hline
\end{tabular}

For SPT available, results represent the number of subjects who were skin prick tested (as a percentage of the subjects seen at each assessment). $P$-values were from two-sided, two-sample proportion test comparing male and females. Asthma was defined as a doctor's diagnosis of asthma plus either wheeze asthma medication use in the previous 12 months. Eczema was defined as ever eczema plus an itchy rash during the previous 12 months. Rhinitis was problems with sneezing, a runny or blocked nose in the absence of cold or flu in the previous 12 months.
Table 2. Percentage of participants with asthma, eczema and rhinitis at each visit according to SPT results at that visit

\begin{tabular}{|c|c|c|c|c|}
\hline & & SPT positive & SPT negative & $P$-value \\
\hline \multirow[t]{6}{*}{ Asthma } & At & $36.27 \%$ (70/193) & $11.44 \%(90 / 787)$ & $<0.0001$ \\
\hline & 4 years & & & \\
\hline & At & $35.74 \%$ (99/277) & $8.59 \%(65 / 757)$ & $<0.0001$ \\
\hline & 10 years & & & \\
\hline & At & $32.19 \%(113 / 351)$ & $9.98 \%(50 / 501)$ & $<0.0001$ \\
\hline & 18 years & & & \\
\hline \multirow[t]{6}{*}{ Eczema } & At & 26.94\% (52/193) & $9.02 \%(71 / 787)$ & $<0.0001$ \\
\hline & 4 years & & & \\
\hline & At & $22.02 \%(61 / 277)$ & $12.93 \%(98 / 758)$ & $<0.0001$ \\
\hline & 10 years & & & \\
\hline & At & $15.91 \%(56 / 352)$ & $9.78 \%(49 / 501)$ & 0.0081 \\
\hline & 18 years & & & \\
\hline \multirow[t]{6}{*}{ Rhinitis } & At & $17.10 \%(33 / 193)$ & $3.44 \%$ (27/786) & $<0.0001$ \\
\hline & 4 years & & & \\
\hline & At & 49.46\% (137/277) & $17.39 \%(132 / 759)$ & $<0.0001$ \\
\hline & 10 years & & & \\
\hline & At & $65.91 \%(232 / 352)$ & $20.36 \%(102 / 501)$ & $<0.0001$ \\
\hline & 18 years & & & \\
\hline
\end{tabular}

Number percentage of participants with each disease out all the participants with positive or negative SPT, depending on the column. $P$-values represent chi-squared tests comparing the percentage of each disease in those with positive and negative SPT results. Asthma, eczema and rhinitis were defined as per Table 1 .

generally showed a higher prevalence of sensitization than females although not statistically significant at age 4 after correction for multiple testing (Table 3). The proportion sensitized to any allergen significantly increased with age (average annual increase of 7\%, $P<0.001)$. This increasing sensitization with age was significantly greater in males than in females $(P=0.001)$.

\section{Incidence and remission of sensitization across childhood and adolescence}

Positive and negative transitions were compared, in a similar way, between the sexes and across time (Fig. 1). Although the significance weakens after adjusting for multiple testing, males tended to be more likely than females to change from being non-sensitized to sensitized (positive transition) during each observation period $(P<0.03$ for each, two-sample proportion test) while females were more likely than males to outgrow their sensitization (negative transition) after 10 years of age $(P=0.044)$. Positive transition from non-sensitization to sensitization was found to increase significantly across childhood and adolescence $(P<0.001)$. For negative transition from sensitized to non-sensitized, there was a significant reduction with increasing age for both sexes combined $(P<0.001)$ and each individual sex (males: $P=0.001$; females: $P=0.015$ ). There was no significant sex-time interaction. This suggests 
Table 3. Prevalence of sensitization over childhood and adolescence

\begin{tabular}{|c|c|c|c|c|}
\hline Point prevalence \% ( $n /$ total) & 4 years & 10 years & 18 years & $\begin{array}{l}\text { Percentage increase in } \\
\text { prevalence per year } \\
\text { of age }(P \text {-value })^{\dagger}\end{array}$ \\
\hline \multicolumn{5}{|l|}{ Sensitization to any allergen } \\
\hline Both & $19.7 \%(193 / 980)$ & $26.9 \%(279 / 1036)$ & $41.3 \%(352 / 853)$ & $7 \%(<0.001)$ \\
\hline Males & $22.7 \%(112 / 493)$ & $31.0 \%(160 / 516)$ & $47.7 \%(194 / 407)$ & $8 \%(<0.001)$ \\
\hline Females & $16.6 \%(81 / 487)$ & $22.9 \%(119 / 520)$ & $35.4 \%(158 / 446)$ & $5 \%(0.001)$ \\
\hline$P$-value for difference in prevalence by sex at each age* & 0.016 & 0.003 & 0.001 & \\
\hline \multicolumn{5}{|c|}{ interaction between sex and age of assessment $P$-value 0.001} \\
\hline \multicolumn{5}{|l|}{ Sensitization to outdoor allergens } \\
\hline Grass pollen & $7.75 \%(76 / 980)$ & $12.93 \%(134 / 1036)$ & $23.85 \%(203 / 851)$ & $8 \%(<0.001)$ \\
\hline Tree pollen & & $1.84 \%(19 / 1035)$ & $5.87 \%(50 / 852)$ & $15 \%(<0.001)$ \\
\hline \multicolumn{5}{|l|}{ Sensitization to indoor allergens } \\
\hline Cats & $5.81 \%(57 / 980)$ & $7.92 \%(82 / 1035)$ & $11.74 \%(100 / 852)$ & $5 \%(<0.001)$ \\
\hline Dogs & $2.45 \%(24 / 980)$ & $4.44 \%(46 / 1035)$ & $10.56 \%(90 / 852)$ & $11 \%(<0.001)$ \\
\hline House dust mite & 11.93\% (117/980) & 19.23\% (199/1035) & $28.91 \%(246 / 851)$ & $6 \%(<0.001)$ \\
\hline Alternaria & $4.80 \%(47 / 980)$ & $2.13 \%(22 / 1035)$ & $8.10 \%(69 / 852)$ & $5 \%(<0.001)$ \\
\hline Cladosporium & $2.14 \%(21 / 980)$ & $1.06 \%(11 / 1035)$ & $3.05 \%(26 / 852)$ & $4 \%(0.12)$ \\
\hline \multicolumn{5}{|l|}{ Sensitization to food allergens } \\
\hline Cod & $0.71 \%(6 / 843)$ & $0.77 \%(8 / 1034)$ & $1.88 \%(16 / 852)$ & 9\% (0.028) \\
\hline Peanut & $1.12 \%(11 / 978)$ & $1.74 \%(18 / 1034)$ & $6.47 \%(55 / 850)$ & $15 \%(<0.001)$ \\
\hline Egg & $0.82 \%(8 / 980)$ & $0.39 \%(4 / 1034)$ & $0.94 \%(8 / 851)$ & $2 \%(0.7)$ \\
\hline Milk & $1.43 \%(14 / 980)$ & $0.29 \%(3 / 1034)$ & $1.17 \%(10 / 852)$ & $-2 \%(0.71)$ \\
\hline Soya & $0.20 \%(2 / 979)$ & $0.19 \%(2 / 1034)$ & $0.71 \%(6 / 850)$ & $11 \%(0.12)$ \\
\hline
\end{tabular}

${ }^{*} P$-value (two-sided) represents the significance of the difference in prevalence between males and females at each assessment using two-sample test on proportions.

†Percentage increase in prevalence per year of age from 4 to 18 years using a GEE analysis $(P$-value); the increase in prevalence in each year is calculated as the percentage of the prevalence in the previous year.

${ }^{\ddagger} P$-value for the sex-time interaction, which represents the difference between males and females across time in terms of the change of prevalence using a GEE analysis.

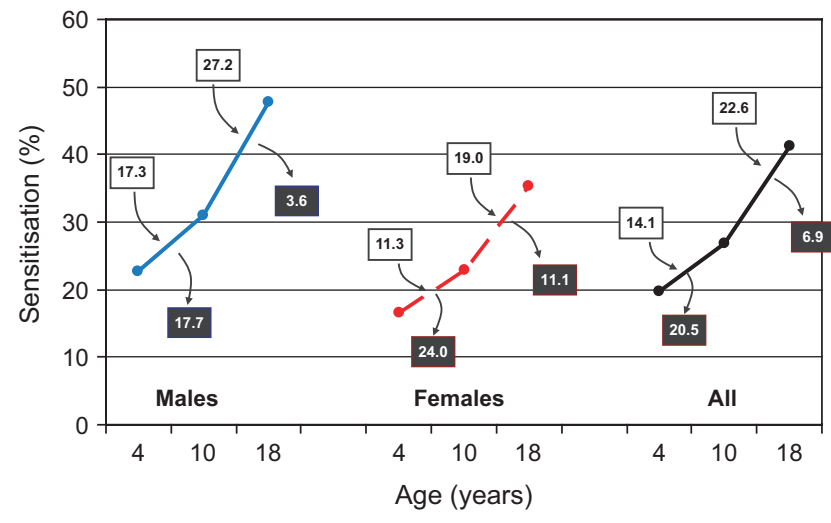

Fig. 1. Prevalence of sensitization across childhood and adolescence. Lines represent the prevalence of sensitization at each assessment for boys (blue) and girls (red). Figures in open boxes represent percentage of population without atopy who developed it during that period. Figures in closed boxes represent percentage of those with atopy who outgrew it during that period.

that increasing numbers of both males and, to a lesser extent, females develop sensitization over childhood and adolescence with females tending to be more likely than males to outgrow their sensitization.

\section{The pattern of sensitization across childhood and adolescence}

To understand how sensitization changes across childhood and adolescence at the level of individuals, we performed a configural frequency analysis (Table 4). Males and females had similar patterns of sensitization across childhood and adolescence. In general, once a child became sensitized at age 4 years, they remain sensitized as indicated by the significant over-representation of the PPP pattern and the significant under-representation of PPN, PNP and PNN. Furthermore, if they were not sensitized by 10 years, they tended to stay non-sensitized as revealed by the significant over-representation of the NNN and significant under-representation of NPN and NNP patterns. It was also apparent that some children outgrew their sensitization (PPN or PNN patterns). The configural frequency analysis was also performed separately for food, indoor and outdoor allergens (Table 4). The test results were consistent between the different sexes and both their patterns were in agreement with the overall pattern (results not shown). A simple comparison between different allergens revealed a similar pattern of results to sensitization to any allergen 


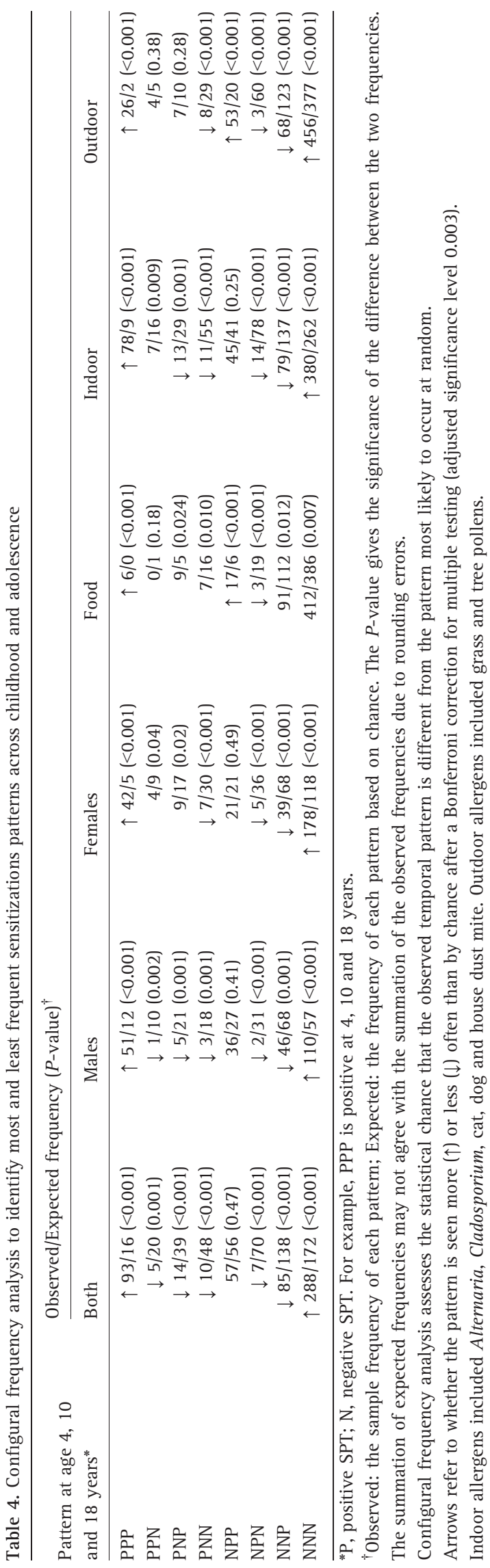

with one important exception. There was a subgroup of children who developed new sensitization to outdoor or food allergens after 4 years of age, given the significance of the NPP pattern, when they typically became sensitized to grass pollen or peanut.

To understand the pattern of co-sensitization to different allergens at the level of individual participants at each assessment, we performed a cluster analysis on data from each assessment (Table 5). Children were excluded from the analysis if there was no record across all the three assessment times, which gave us a total of 1242 records at each assessment. The most important cluster of sensitization at age 4 was characterized by a predominance of house dust mite (HDM) sensitization. This pattern was increasingly prevalent at 10 and 18 years. A second cluster included children who were predominately sensitized to grass pollen, seen most clearly from the age of 10 years. Some children in this cluster were additionally sensitized to other allergens such as cat, dog, HDM and peanut.

How individual participants transition between each of the cluster analysis derived patterns of co-sensitization at 4 and 10 years of age is shown in Fig. 2. It demonstrates that most of the non-sensitized children at age $4 \quad(n=802)$ remained non-sensitized at age 10 years. Of the children with predominantly HDM $(n=40)$ sensitization at age 4 years, about half develop polysensitization to indoor and outdoor allergens at 10 years of age (Fig. 2). Focusing on children at 10 years with no sensitization, just under half went on to develop sensitization to at least one allergen at age 18. Of the children with only HDM sensitization at age $10(n=98)$, most continued to be mono-sensitized to HDM at age 18 years. Between 10 and 18 years, the cluster characterized by grass pollen sensitization $(n=20)$ was relatively stable, whereas children with polysensitization to grass pollen, cat and HDM at age 10 years transitioned to a variety of polysensitized groups at age 18 years.

\section{Discussion}

Skin prick testing was performed on the majority of participants in the 1989 Isle of Wight birth cohort at 4, 10 and 18 years of age. The prevalence of sensitization to any allergen increased over childhood and adolescence until 41.3\% was affected at 18 years. Some however did outgrow their allergic sensitization. Boys were always more likely to be sensitized than girls. Sensitization to individual aeroallergens and peanuts also increased over this period. Despite this, sensitization status at age 4 years usually predicted later status. The patterns of sensitization to different allergens changed across childhood with individuals becoming increasingly polysensitized. 


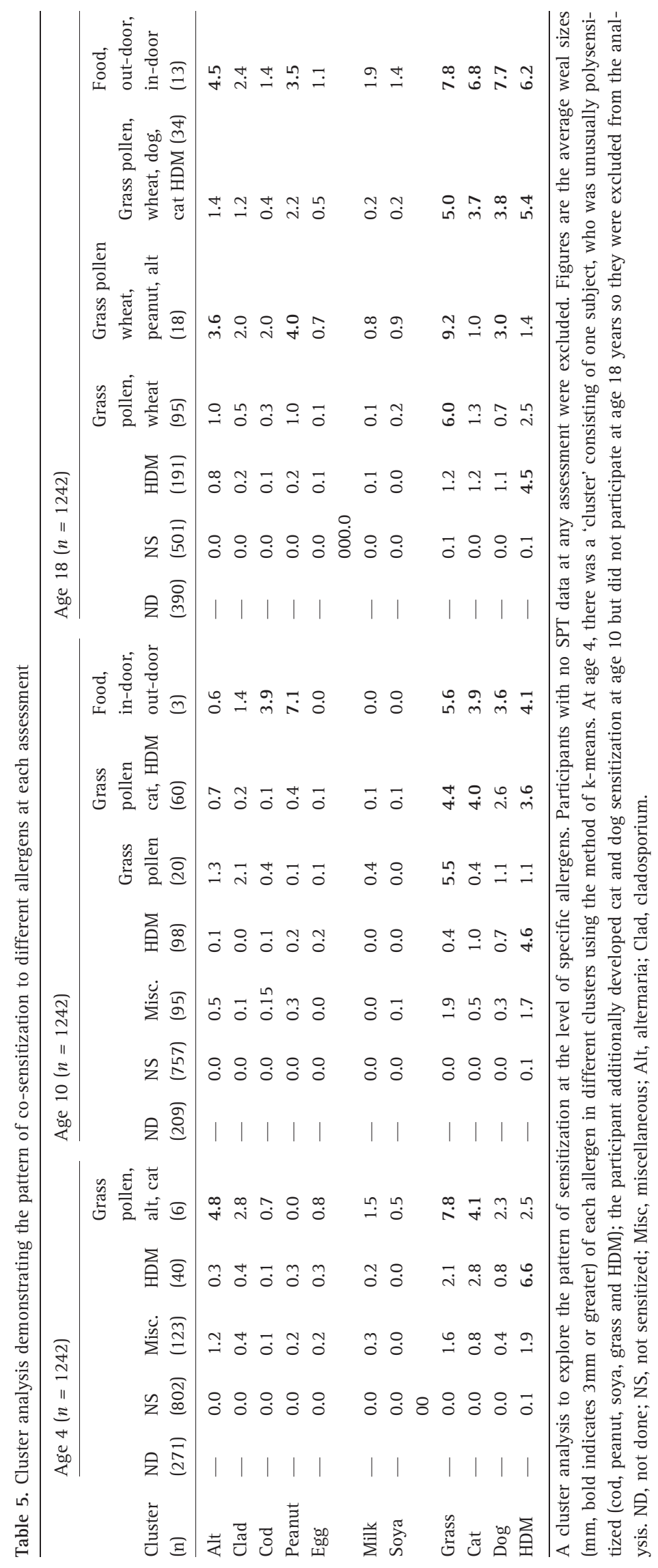




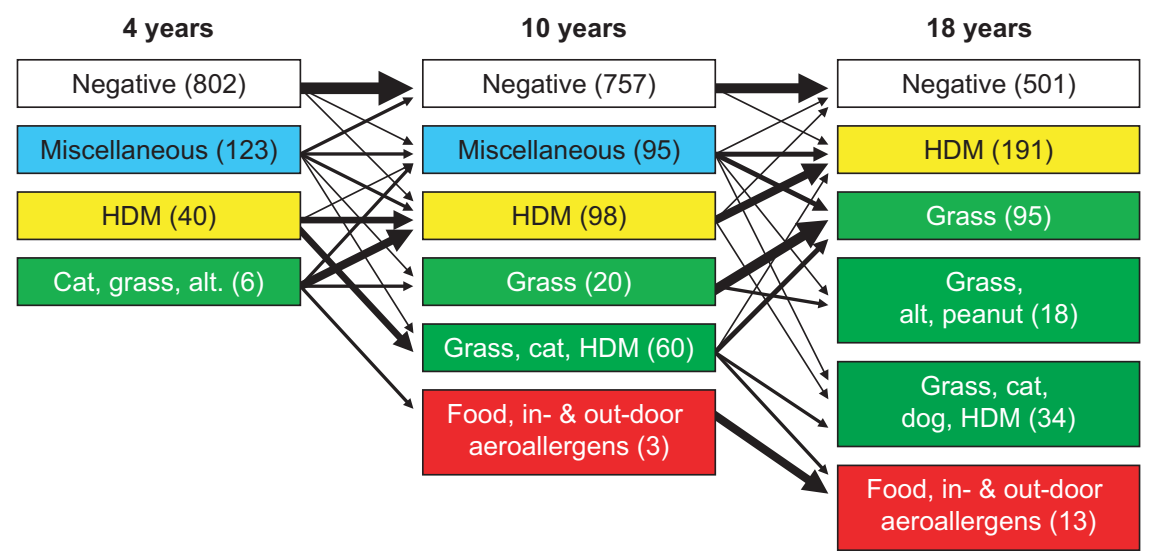

Fig. 2. Patterns of transitions between sensitization clusters from 4 to 18 years. Boxes present clusters of sensitization at each age. Numbers in brackets represent numbers of subjects in each cluster. Not all subjects are represented in the figure as not all were allocated to one of these clusters. Width of arrows represents the probability of transition from one cluster to another between successive assessment time-points. Alt: alternaria. Only transition probabilities $\geq 5 \%$ included. Key allergens in the food group were peanut and cod. Indoor aeroallergens were HDM, cat and dog, while outdoor aeroallergens were grass pollen and alternaria.

The Isle of Wight cohort has one of the highest prevalence of sensitization of any European birth cohort. For example, the prevalence in the Avon Longitudinal Survey of Pregnancy and Childhood was only 15\% at 6 years [26], while a Swedish cohort recorded rates of $30 \%$ at 7-8 years [19]. Other cohorts have also shown increasing levels of sensitization over childhood and adolescence $[4,17,19,27]$. One potential explanation is a general increasing reactivity of the skin over childhood as demonstrated by the increasing response to histamine [28]. The SPT guidelines do however suggest a fixed cut-off diameter for a positive SPT test across childhood [5]. The persistence of sensitization seen at age 4 years suggests that the atopic pathway is set early on in life. This is likely to have relevance towards clinical disease as it had been shown that early allergic sensitization is an important predictor for persistent allergic disease with associated morbidity [1, 4, 29]. Still only a proportion of children with allergic sensitization express clinical allergy. Importantly, there were small but significant number of individuals (Table 4), more likely to be females than males during adolescence, who did outgrow their sensitization confirming that allergic sensitization is not a completely stable phenotype [17].

Individual children in the cohort were sensitized to a wide range of allergens although the most prevalent were HDM, grass pollen and cat, similar to other UK data [26]. Geographical differences are seen, for example, in the Swedish BAMSE Birth Cohort the most prevalent allergens were birch and grass pollens closely followed by cat and dog with little HDM sensitization [17]. This demonstrates the need to understand the local sensitization pattern when clinically assessing children and teenagers in clinic [30].
Our data suggest that there are specific patterns of co-sensitization across childhood and adolescence. At 4 years of age, the largest cluster was one sensitized to HDM. As these 4-year olds grow up, there was a tendency for them to become more sensitized. For example, a third of the HDM group became additionally sensitized to grass pollen and cat at age 10 years (Fig. 2). At age 18 years, there was a further increase in the number of polysensitized groups. This progression occurs due to the development of sensitization to new allergens and to the development of sensitization to related allergens. It is possible that some of the increased sensitization to peanut at 18 years (Table 1) was also due to primary sensitization to grass or birch pollen [31]. This illustrates one of the problems of using whole allergens for allergy testing and how component resolved diagnosis may help us to distinguish true sensitization from cross-sensitization in the future [32]. All these pollen sensitized young adults are also at risk of developing secondary food allergies, pollen-food allergy syndrome [33].

The strengths of this study are the high proportions of follow-up achieved to 18 years of age. With increasing age, less were skin prick tested due to the increasing number of assessments that were undertaken by telephone. However, even at 18 years, 65\% of participants had SPT. This minimizes the potential for bias and supports the generalizability of the results. Furthermore, we have demonstrated that there is no allergic disease based bias in terms of who were tested with skin prick testing at each assessment. A potential weakness is that there may have been subtle changes in the SPT solutions over the 20 years of the study. This might explain the lower prevalence of milk and egg sensitization at 10 compared to 4 and 
18 years (Table 3 ). We did, however, use the same SPT procedure throughout the study with key researchers involved in all assessments and the change in prevalence of sensitization is considerable with increasing age. Also asthma, eczema and rhinitis were reported by parents. Accurately defining asthma is particularly challenging in the pre-school age group even when a doctor's diagnosis is included in the definition. It is interesting to note that the rate of asthma in this cohort is similar through childhood and adolescence (Table 1), with children outgrowing their asthma being replace by others developing it [10].

These findings suggest that sensitization continues to increase over childhood and into adolescence with over $40 \%$ of teenagers being affected by 18 years of age. Sensitization generally starts with one allergen but progresses towards polysensitization in many individuals as they move through adolescence. This changing pattern of sensitization over childhood and adolescence needs to be better characterized and its relevance to clinical disease needs to be established. Clinically, it suggests that allergic patients should be regularly reassessed to detect any additional, clinically relevant allergic sensitizations or in some cases loss of sensitization. While for most, sensitization is a permanent event, some individuals do seem to outgrow this and we need to understand why this occurs as it may have important implications for modifying the natural history of sensitization and allergic disease. Future investigations need to determine how family history of allergy and environmental factors may influence the different patterns of sensitization and whether these patterns are closely associated with specific clinical manifestations of clinical allergy [34].

\section{Acknowledgements}

The 18-year assessment of the 1989/90 Isle of Wight birth cohort was funded by the National Institute of Health, USA (R01 HL082925-02). ALK-Abello kindly provided the skin prick testing reagents for the cohort assessment, but they had no input into the study design or analysis of the data presented in this manuscript. We acknowledge the help of all the staff at The David Hide Asthma and Allergy Research Centre in undertaking the 18-year and previous assessments of 1989/90 Isle of Wight birth cohort and Stephen Potter for drawing the figures. We also acknowledge the help of the study subjects and their families who have participated in this project over the last two decades.

Statement of contribution by authors: GR conceived the idea and with HZ developed the analysis plan. HZ undertook the data analysis. All the authors contributed to the review of the results. GR drafted the manuscript and all the authors were involved revising the draft into the final manuscript.

Conflicts of interests: None of the authors have any conflict of interests with this manuscript.

\section{References}

1 Kurukulaaratchy RJ, Matthews S, Arshad SH. Defining childhood atopic phenotypes to investigate the association of atopic sensitisation with allergic disease. Allergy 2005; 60:1280-6.

2 Asher MI, Montefort S, Bjorksten B et al., ISAAC Phase Three Study Group. Worldwide time trends in the prevalence of symptoms of asthma, allergic rhinoconjunctivitis, and eczema in childhood: ISAAC phases one and three repeat multicountry cross-sectional surveys. Lancet 2007; 370:1128.

3 Gupta R, Sheikh A, Strachan DP, Anderson HR. Time trends in allergic disorders in the UK. Thorax 2007; 62:91-6.

4 Edgecombe K, Latter S, Peters S, Roberts G. Health experiences of teenagers with uncontrolled severe asthma: interaction with health care professionals and concordance with therapy. Arch Dis Child 2010; 95:985-91.
5 Dreborg S, Frew A. Position paper: allergen standardization and skin tests. Allergy 1993; 48:49-54.

6 Sadeghnejad A, Karmaus W, Davis S, Kurukulaaratchy RJ, Matthews S, Arshad SH. Raised cord serum immunoglobulin increases the risk of allergic senstisation at ages 4 and 10 and asthma age 10. Thorax 2004; 59:936-42.

7 Allam JP, Zivanovic 0, Berg C, Gembruch $\mathrm{U}$, Bieber T, Novak N. In search for predictive factors for atopy in human cord blood. Allergy 2005; 60:743-50.

8 Sly PD, Boner AL, Bjorksten B et al. Early identification of atopy in the prediction of persistent asthma in children. Lancet 2008; 372:1100-6.

9 Arshad SH, Kurukulaaratchy RJ, Fenn M, Matthews S. Early life risk factors for current wheeze, asthma and bronchial hyperresponsiveness at 10 years of age. Chest 2005; 127:502-8.

10 Kurukulaaratchy RJ, Raza A, Scott M et al. Characterisation of asthma that develops during adolescence; findings from the Isle of Wight Birth Cohort. Respir Med 2012; 106:329-37.

11 Kurukulaaratchy R, Karmaus W, Raza A, Matthews S, Roberts G, Arshad SH. The influence of gender and atopy on the natural history of rhinitis in the first 18 years of life. Clin Exp Allergy 2011; 41:851-9.

12 Marinho S, Simpson A, Soderstom L, Woodcock A, Ahlstedt S, Custovic A. Quantification of atopy and the probability of rhinitis in preschool children: a population-based birth cohort study. Allergy 2007; 62:1379-86.

13 Roberts G, Lack G. Diagnosing peanut allergy with skin prick and specific IgE testing. J Allergy Clin Immunol 2005; 115:1291-6.

14 DunnGalvin A, Daly D, Cullinane C et al. Highly accurate prediction of food challenge outcome using routinely available clinical data. J Allergy Clin Immunol 2011; 127:633-9.

15 Scott M, Raza A, Karmaus W et al. The influence of atopy and asthma on exhaled nitric oxide in an unselected 
birth cohort study. Thorax 2010; 65:258-62.

16 Carroll WD, Lenney W, Child F et al. Asthma severity and atopy: how clear is the relationship? Arch Dis Child 2006; 91:405-9.

17 Asarnoj A, Ostblom E, Kull I et al. Sensitization to inhalant allergens between 4 and 8 years of age is a dynamic process: results from the BAMSE birth cohort. Clin Exp Allergy 2008; 38:1507-13.

18 Dottorini ML, Bruni B, Peccini $\mathrm{F}$ et al. Skin prick-test reactivity to aeroallergens and allergic symptoms in an urban population of central Italy: a longitudinal study. Clin Exp Allergy 2007; 37:188-96.

19 Ronmark E, Bjerg A, Perzanowski M, Thomas Platts-Mills T, Lundback B. Major increase in allergic sensitization in schoolchildren from 1996 to 2006 in northern Sweden. J Allergy Clin Immunol 2009; 124:357-63.

20 Matricardi PM, Bockelbrink A, Gruber C et al. Longitudinal trends of total and allergen-specific IgE throughout childhood. Allergy 2009; 64:1093-8.

21 Arshad SH, Hide DW. Effect of environmental factors on the development of allergic disorders in infancy. $J$ Allergy Clin Immunol 1992; 90:235-41.

22 Arshad SH, Stevens M, Hide DW. The effect of genetic and environmental factors on the prevalence of allergic disorders at the age of two years. Clin Exp Allergy 1993; 23:504-11.

23 Tariq S, Matthews S, Stevens M, Hakim E, Arshad SH. The prevalence of and risk factors for atopy in early childhood: a whole population birth cohort study. J Allergy Clin Immunol 1998; 101:587-93.

24 Zeger SL, Liang KY. Longitudinal data analysis for discrete and continuous outcomes. Biometrics 1986; 42:121-30.

25 von Eye A. Introduction to configural frequency analysis: the search for type and antitypes in cross-classifications. Cambridge: Cambridge University Press, 1990.

26 Roberts G, Wilman C, Lack G, Henderson J, Strachan D, Golding J. The relationship between aeroallergen and food allergen sensitization in childhood. Clin Exp Allergy 2005; 35:299300.

27 Venter C, Pereira B, Grundy J et al. Incidence of parentally reported and clinically diagnosed food hypersensitivity in the first year of life. J Allergy Clin Immunol 2006; 117:1118-24.

28 Ronchetti R, Pia Villa M, Ciofetta G, Barreto M, Falasca C, Martella S. Changes over 13 years in skin reactivity to histamine in cohorts of children aged 9-13 year. Allergy 2001; 56:43641.
29 Kurukulaaratchy RJ, Matthews S, Arshad SH. Relationship between childhood atopy and wheeze: what mediates wheezing in atopic phenotypes? Ann Allergy Asthma Immunol 2006; 97:84-91.

30 Heinzerling L, Frew AJ, Bindslev-Jensen C et al. Standard skin prick testing and sensitization to inhalant allergens across Europe - a survey from the GA2LEN network. Allergy 2005; 60:1287-300.

31 Nicolaou N, Poorafshar M, Murray C et al. Prevalence and differentiation using component-resolved diagnostics. $J$ Allergy Clin Immunol 2010; 125: 191-7.

32 Constantin C, Quirce S, Poorafshar M et al. Micro-arrayed wheat seed and grass pollen allergens for componentresolved diagnosis. Allergy 2009; 64:1030-7.

33 De Knop KJ, Verweij MM, Grimmelikhuijsen $\mathrm{M}$ et al. Age-related sensitization profiles for hazelnut (Corylus avellana) in a birch-endemic region. Pediatr Allergy Immunol 2011; 22: e139-49.

34 Arshad SH, Karmaus W, Raza A et al. The effect of parental risk on childhood allergy depends on the sex of the child. J Allergy Clin Immunol 2012; 130:427-34. 